\title{
FIGO Stage 0
}

National Cancer Institute

\section{Source}

National Cancer Institute. FlGO Stage O. NCI Thesaurus. Code C96243.

A FIGO stage term that applies to gynecologic cancers and refers to carcinoma in situ.

For cervical cancer, it used to refer to cancer that is confined to the cervical epithelium without stromal invasion; for endometrial cancer, it used to refer to cancer that is confined to the endometrium without myometrial invasion. FIGO no longer includes stage 0 cervical or endometrial cancer. 